Vol. 15, Núm. 4

Oct.-Dic. 2019. pp 206-213 doi: $10.35366 / 94046$

https://dx.doi.org/10.35366/94046

\title{
Aflojamiento aséptico en la prótesis total de cadera
}

\author{
Aseptic loosening in the total hip prosthesis
}

Vicente Reyes de la Parra*

\begin{abstract}
* Cirujano Ortopedista, Subespecialidad en Cirugía Reconstrucción de Rodilla-Cadera, Hospital Ángeles Xalapa. Profesor invitado curso Especialidad Ortopedia, Universidad Veracruzana, Centro de Alta Especialidad, Veracruz. Profesor titular curso Patología Quirúrgica en la Facultad de Medicina de la Universidad Anáhuac, Xalapa.
\end{abstract}

Correspondencia: Dr. Vicente Reyes de la Parra. Hospital Ángeles Xalapa. Carretera Xalapa-Veracruz No. 560, consultorio 820, Col. Pastoresa, 91198, Xalapa, Veracruz. Correo electrónico: drvicentereyes@gmail.com

\section{RESUMEN}

El aflojamiento aséptico y osteólisis es actualmente la tercera causa de falla e indicación para revisión en la artroplastia total de cadera. El mejor entendimiento de la fisiopatología, las causas y las formas de prevención de la osteólisis han disminuido significativamente su prevalencia durante los últimos 20 años. Hoy en día $10-15 \%$ de las cirugías ATC de revisión tienen como indicación el AA y osteólisis, de estas revisiones $41 \%$ de los casos requieren revisión de todos los componentes, y $50 \%$ de los casos requieren sólo recambio de alguno de los tres componentes (13-17\% componente femoral, $13-17 \%$ componente acetabular y $13-17 \%$ cambio de cabeza femoral-liner acetabular). La mayoría de los casos de AA son asintomáticos hasta la falla total del implante. En todos los casos de aflojamiento es obligatorio descartar que no se trate de un aflojamiento séptico. La tomografía computarizada es el método ideal para clasificar el grado de osteólisis, tanto femoral como acetabular, y para una correcta planificación preoperatoria. El tratamiento médico para la osteólisis en ATC tiene como objetivo sólo detener la progresión, mejorar la función y disminuir el dolor; sin embargo, una vez presentado el cuadro sintomático y confirmado el diagnóstico de AA por imagen, la cirugía

\section{ABSTRACT}

Aseptic loosening and osteolysis is the third leading indication for revision total hip arthroplasty. Better understanding of the pathophysiology of osteolysis, causes and preventive measures have significantly decreased ALA prevalence during the past 20 years. Currently $10-15 \%$ of revision THR are performed due to AL. From those revisions, $59 \%$ require revision of all components, while $41 \%$ remaining only require revision of one of them: $13 \%$ femoral component, $16 \%$ acetabular component and $12 \%$ femoral head and linner. The majority of $A L$ are asymptomatic until implant subsidence. In all AL cases, septic loosening must be ruled out. Usually normal ESR and $C$ react protein is enough to rule out infection, although elevated levels obligate further infection work up. Computed tomography is the ideal method to assess and classify osteolysis, and for revision surgical planning. Medical treatment for osteolysis in THR is intended to stop AL progression, reduce pain and increased activity and function. However, once symptoms develop and diagnosis of $A L$ is confirmed, revision surgery is the treatment of choice. Revision can be performed in one or all components, depending of loosening. Bone allograft 
de revisión es el tratamiento de elección. La cirugía de ATC de revisión puede ser de sólo un componente, con evidencia de buenos resultados conservando componentes sin aflojamiento, o puede realizarse recambio total de componentes. El uso de aloinjerto otorga beneficios para restablecer el stock óseo; sin embargo, el uso de aumentos metálicos tiene la ventaja de apoyo y rehabilitación inmediatos.

Palabras clave: ATC (artroplastia total de cadera), AA (aflojamiento aséptico), osteólisis, polietileno de ultra alto peso molecular con enlaces cruzados UHMWXLP. can help restore bone stock, but metallic augments have the advantage in regards to allowing immediate weight bearing, and earlier physical therapy.

Keywords: THR (total hip arthroplasty), aseptic loosening, osteolysis, ultra high molecular weight cross link polywethilene UHMWXLP.

\section{INTRODUCCIÓN}

La artroplastia total de cadera es el procedimiento quirúrgico ortopédico de mayor éxito en los últimos 50 años. ${ }^{1,2}$ La curva de edad población de nuestro país y el aumento en la expectativa de vida hacen que la necesidad de practicar una ATC de revisión sea cada vez más frecuente.

Considerando las estadísticas actuales respecto a la curva edad población, expectativa de vida así como índices de obesidad ${ }^{3-5}$ obligan a mejorar más aún en todos los aspectos los resultados en la artroplastia total de cadera, aumentando la supervivencia de los implantes por un lado, y también a partir de la ATC primaria a considerar una posible revisión en el futuro.

Entre las causas de revisión para una ATC, el aflojamiento aséptico (AA) y la osteólisis se ubican en tercer lugar como indicación para una cirugía de revisión, sólo después de inestabilidad-luxación e infección. ${ }^{6}$

Hoy en día se sabe que la llamada inicialmente «enfermedad del cemento» es una cadena de eventos biológicos que ocasionan como resultado la osteólisis y en consecuencia el aflojamiento aséptico., ${ }^{3,7,8}$

\section{FISIOPATOLOGÍA DE LA OSTEÓLISIS EN ATC}

La que originalmente fue llamada "enfermedad del cemento", ahora se sabe que es un proceso dinámico en el que osteoclastos activados van fagocitando micropartículas debris, el cual puede ser del polimetilmetacrilato, polietileno o partículas metálicas. . $^{7-10}$

El estímulo fundamental para la osteólisis es la formación de micropartículas por desgaste en la ATC. El micromovimiento generado en la superficie de carga-soporte de la ATC permite la producción de líquido con alto contenido de partículas. ${ }^{7,8,10}$

La generación de partículas en la ATC puede obedecer a dos procesos: desgaste y corrosión. El desgaste ha sido descrito como pérdida de material de la prótesis 
en forma de micropartículas debris. Corrosión se define como un proceso electroquímico en el que iones metálicos son liberados de la superficie del implante. ${ }^{3}$

Se han descrito cuatro modalidades para el desgaste. Modalidad 1 se refiere a la generación de partículas que ocurre entre el movimiento de dos superficies de carga-soporte que fueron diseñadas para ello en su manufactura. Modalidad 2 se refiere a alguna de las superficies de carga-soporte que fricciona contra una superficie secundaria no diseñada para carga-soporte. Modalidad 3 se refiere a un cuerpo extraño interpuesto entre las superficies naturales de cargasoporte. Modalidad 4 se refiere a la fricción entre dos superficies secundarias no diseñadas para carga-soporte. .,11,12 $^{8}$

Actualmente se sabe que el mecanismo principal para la osteólisis y aflojamiento aséptico en la ATC es la reacción biológica secundaria al debris generado por el implante. ${ }^{4,7,9}$ El proceso inicia con la fagocitosis mediada por macrófagos de las micropartículas. El tamaño de las micropartículas (más que el tipo de micropartículas) es lo que más osteólisis genera en la reacción biológica osteoclástica.

Partículas menores de 7 micras son fagocitadas por macrófagos y favorecen la liberación de interleucinas 1,6 y del factor necrosis tumoral activando la cascada inflamatoria, mientras que en partículas superiores a 7 micras se ha demostrado que no pueden ser fagocitadas por osteoclastos.

La activación de los macrófagos y osteoblastos provoca una liberación del receptor activador del factor nuclear $\mathrm{Kb}$ ligando, ${ }^{4,7,8}$ el cual se adhiere a receptores de osteoclastos, favoreciendo la reabsorción ósea y formación de granulomas. Sin embargo, la interleucina 6 (IL6) ha demostrado ser el factor inflamatorio más importante en la estimulación del efecto osteoclástico y reabsorción ósea.

\section{DIAGNÓSTICO EN OSTEÓLISIS DE ATC}

Las radiografías simples de pelvis y cadera continúan siendo el método universal para evaluar la evolución postoperatoria de la ATC, ${ }^{5}$ y por lo tanto, el diagnóstico de la osteólisis puede retardarse significativamente, considerando que $40-80 \%$ de los casos iniciales en el aflojamiento aséptico pueden ser asintomáticos y no detectados adecuadamente en radiografías simples. ${ }^{1,13,14}$

La sensibilidad para identificar la osteólisis en radiografías simples puede ser de 50 a $60 \%$, con una especificidad de 70 a $90 \%$. El tiempo promedio en el que lesiones de osteólisis en una ATC pueden hacerse evidentes en las Rxs simples es de 5.7 años. ${ }^{2,13,15}$

En todos los casos de aflojamiento aséptico y osteólisis es obligatorio descartar que no se trate realmente de un aflojamiento séptico. Todo paciente con fístula, o antecedente de infección en zona quirúrgica debe considerarse infectado e iniciar el protocolo de tratamiento aflojamiento séptico. En caso de no tener ninguna evidencia aparente de infección, como protocolo diagnóstico se debe solicitar la velocidad sedimentación globular y proteína $C$ reactiva. ${ }^{10,16}$ Ninguno de estos dos reactantes fase aguda es específico para diagnóstico de infección; sin embargo, su sensibilidad sí es alta (de 90 a 95\%), por lo que niveles normales de ambos pueden descartar infección. En caso de niveles anor- 
malmente elevados nos obliga a realizar aspiración de líquido sinovial y punción de la articulación.

Una vez descartado el proceso infeccioso debe hacerse el diagnóstico y clasificación del AA de ATC, lo cual debe realizarse con estudios de imagen. La AP de pelvis es la proyección inicial de batalla para inicio de diagnóstico. Sin embargo, la técnica tiene gran relevancia para la correcta evaluación de la ATC. La proyección debe centrarse en la sínfisis del pubis, y alinearse con el coxis. Las radiografías laterales de pelvis, lateral del fémur proximal, proyecciones oblicuas tipo Judet tienen utilidad para la valoración de la interfase copa-acetabular y vástago femoral; sin embargo, la tomografía computarizada es el método de elección para determinar el grado de osteólisis, versión de los componentes y pérdida de stock óseo.

Aunque existen diversas clasificaciones para el AA y osteólisis en ATC, la clasificación de Paprosky cumple con ofrecer varias facilidades en diagnóstico y tener relación directa a pronóstico y tratamiento. ${ }^{17,18}$

La clasificación de osteólisis acetabular (Figura 1) está basada en cuatro parámetros: migración del centro de rotación, destrucción o no de la gota de lágrima, osteólisis de isquion e integridad o no de la línea Kohler. La migración central de la copa distingue entre el tipo II cuando es menor de $3 \mathrm{~cm}$ y el tipo III cuando es mayor de $3 \mathrm{~cm}$, y ésta a su vez se subclasifica en subgrupos, considerando principalmente la presencia o no de osteólisis en la gota de lágrima. .,15,18 $^{1}$

La clasificación femoral (Tabla 1) considera la extensión distal de la osteólisis, inicialmente la osteólisis metáfisis femoral proximal (tipos I y II), sin extensión a diáfisis, y osteólisis que involucra diáfisis femoral, siendo el istmo el punto de referencia para diferenciar tipos III A y B. ${ }^{12}$

\section{TRATAMIENTO}

Como primera etapa de tratamiento se sugiere el manejo conservador, principalmente el manejo de la osteólisis periprotésica asintomática como hallazgo
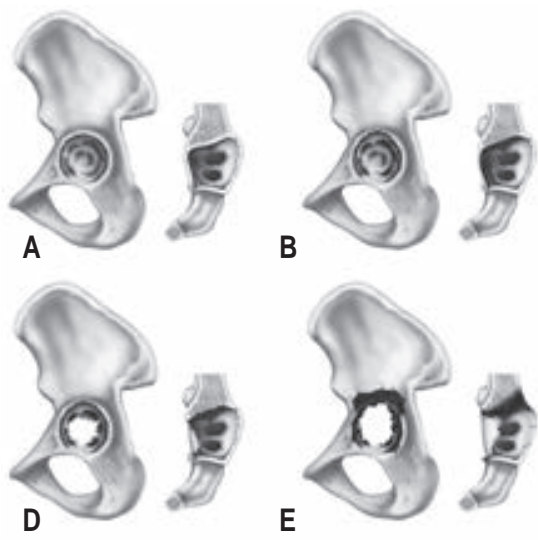

C

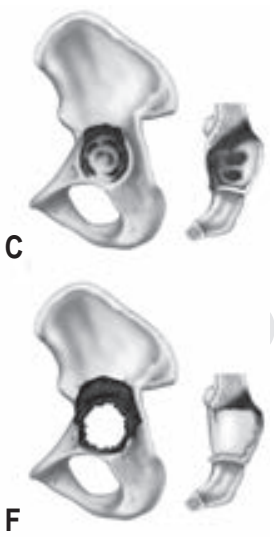

Figura 1:

Clasificación osteólisis acetabular de Paprosky: A) tipo I, B) tipo IIA, C) tipo IIB, D) tipo IIC, E) tipo IIIA, F) tipo IIIC. Tomado de: Sheth NP, Nelson CL, Springer BD, Fehring TK, Paprosky WG. Acetabular bone loss in revision total hip arthroplasty: evaluation and management. J Am Acad Orthop Surg. 2013; 21 (3): 128-139. 
Tabla 1: Clasificación de Paprosky osteólisis femoral.

\begin{tabular}{|c|c|c|c|}
\hline Tipo & Definición & Metáfisis proximal & Diáfisis \\
\hline 1 & Mínima pérdida en metáfisis proximal & Intacta & Intacta \\
\hline ॥ & Pérdida metáfisis proximal moderada a severa & Ausente & Intacta \\
\hline IIIA & $\begin{array}{l}\text { Pérdida ósea extensa y severa en metáfisis proximal } \\
\text { que se extiende hasta diáfisis femoral }\end{array}$ & Ausente & $\begin{array}{l}\text { Menor de } 4 \mathrm{~cm} \\
\text { del istmo }\end{array}$ \\
\hline IIIB & $\begin{array}{l}\text { Pérdida ósea extensa y severa en metáfisis proximal } \\
\text { que se extiende hasta diáfisis femoral }\end{array}$ & Ausente & $\begin{array}{l}\text { Mayor de } 4 \mathrm{~cm} \\
\text { del istmo }\end{array}$ \\
\hline IV & $\begin{array}{l}\text { Pérdida ósea severa completa en metáfisis y } \\
\text { diáfisis femoral }\end{array}$ & Ausente & $\begin{array}{l}\text { Ausente o con } \\
\text { defecto severo }\end{array}$ \\
\hline
\end{tabular}

Modificado de: Sheth NP, Nelson CL, Paprosky WG. Femoral bone loss in revision total hip arthroplasty: evaluation and management. J Am Acad Orthop Surg. 2013; 21 (10): 601-612.

radiográfico en seguimiento de la ATC, se recomienda modificación y disminución de nivel actividades, descarga por medio de bastón o muleta canadiense y AINEs para manejo de síntomas, y se ha propuesto el uso de antirresortivos (bifosfonatos-denosumab) como parte del manejo con evidencia no concluyente respecto a su eficacia.

Se ha propuesto el tratamiento médico para osteólisis en ATC en algunos casos. Si se toma en cuenta que de 20 a $30 \%$ de las ATC tendrán algún grado de osteólisis entre los siete y 14 años, y que de éstos la mayoría son asintomáticos, considerar que el manejo no quirúrgico puede tener mucho sentido. Sin embargo, la evidencia no es aún concluyente respecto al beneficio de medicamentos para manejo de la osteólisis en ATC. Se ha estudiado la eficacia de los bifosfonatos para inhibir la osteólisis, encontrándose que el uso durante un año, de seis a 12 semanas posterior a la implantación de la ATC puede disminuir la incidencia de la osteólisis; sin embargo, en metaanálisis y estudios con nivel evidencia I aún no se ha podido recomendar el uso sistemático de bifosfonatos en la ATC y los efectos adversos de los bifosfonatos en las fracturas atípicas, incluso fracturas periprotésicas que se conocen actualmente, han llevado al abandono general en esta modalidad de tratamiento. ${ }^{19,20}$

El factor nuclear receptor activador transmembrana (RANK) y su ligando correspondiente (RANKL) es un regulador importante de la actividad osteoclástica y reabsorción ósea. El denosumab ha demostrado ser un tratamiento eficiente y seguro para osteoporosis al inhibir bloquear RANKL y por lo tanto, inhibe la actividad osteoclástica. Sin embargo, este beneficio aún requiere comprobarse para inhibir la osteólisis periprotésica, y aunque estudios recientes han reportado resultados favorables, no existe aún ningún estudio controlado con nivel evidencia I-II que recomiende el uso de denosumab para el tratamiento en la osteólisis de la ATC.

Cuando la osteólisis ha provocado un AA de la ATC sintomático con falla del implante, o evidencia radiográfica de progresión en la osteólisis durante el seguimiento, se indica la cirugía de revisión. El diagnóstico y clasificación correcta del AA y osteólisis son indispensables para la planificación en la ATC de revisión. 
Se requiere tomografía computarizada para la correcta clasificación, y definir si la cirugía requiere revisión de todos los componentes de la ATC, o sólo la revisión parcial de la misma. De acuerdo con la bibliografía, estadísticamente en la cirugía de revisión hoy en día se realiza el recambio de todos los componentes y es necesaria en $41 \%$ de los casos, la revisión de componente acetabular $16 \%$, cabeza-liner acetabular $12 \%$ y componente femoral $13 \%$ aisladas, respectivamente..$^{6,11,14,17,21}$

Para la revisión en componente femoral, principalmente se cuenta con tres opciones: copas jumbo no cementadas, aloinjerto para impactación o en segmento osteocondral estructural y el uso de cuñas metálicas y anillos reforzamiento. ${ }^{22-24}$

El uso de aloinjerto impactado o estructural, tiene la ventaja de mejorar el stock óseo acetabular, y es el método de reconstrucción que se elige en pacientes jóvenes, en quienes posiblemente se requiera una futura revisión. Sin embargo, los resultados en el uso de aloinjerto han sido variables respecto a su integración, y el subsecuente aflojamiento. ${ }^{25,26}$

El uso de copas jumbo con cuñas metálicas para la solución de defectos osteolíticos acetabulares es actualmente la forma de revisión más empleada por cirujanos de cadera en cirugía ATC de revisión. ${ }^{27}$ Los resultados han sido más constantes, eliminando el riesgo de reabsorción ósea en injertos y disminuyendo el riesgo de aflojamiento o falla en implantes con tasas de supervivencia de copas revisión de más de 90 a $95 \%$ a cinco años en diferentes series. ${ }^{22,25}$

En ocasiones el uso de copas oblongas es necesario para la integración y adaptación a zonas de osteólisis acetabular; sin embargo, en ocasiones es necesario el uso de copas «triflanco» hechas a medida con modelos realizados con impresoras tridimensionales para resolver defectos acetabulares, principalmente Paproskys III A y B.

En la revisión del componente femoral, el uso de vástagos de revisión no cementados es el estándar de oro para resolver la gran mayoría de los casos. La longitud y diseño del vástago dependerá del grado de osteólisis de acuerdo con la clasificación Paprosky, pero la gran mayoría de las revisiones pueden resolverse con vástagos de longitud de $180 \mathrm{~mm}$. Los vástagos cementados de revisión han ido cayendo en desuso, ya que en la actualidad existen pocas indicaciones y el diseño actual de vástagos modulares no cementados con fijación diafisiaria distal a zonas de osteólisis, con longitudes disponibles desde 210, 240 hasta $270 \mathrm{~mm}$ en el mercado permiten resolver el recambio y revisión femoral sin necesidad del cementado. ${ }^{12,21,25}$

La extracción de un vástago femoral puede resultar un reto, aun con aflojamiento aséptico y osteólisis, y principalmente en caso de no presentarlo, pero que por el diseño, el caso en particular del acetábulo, sea necesario el recambio de ambos componentes, por lo que debe considerarse la osteotomía trocantérica extendida para su retiro, y el vástago de revisión seleccionado debe contemplar dicha osteotomía para su fijación así como el cerclaje-cables posterior.

\section{CONCLUSIONES}

La ATC es hasta el momento la cirugía ortopédica más exitosa. Ha demostrado mejorar significativamente la calidad de vida en pacientes, con una baja tasa 
de complicaciones. Sin embargo, el incremento en la expectativa de vida en la población mundial ha generado la necesidad de desarrollar mejores opciones que aumenten la supervivencia de los implantes en cirugía primaria de cadera y en cirugía de revisión.

El desarrollo de polietilenos con enlaces altamente cruzados ha disminuido de manera significativa la incidencia del AA en la ATC, y los metales ultraporosos con mejor osteointegración, de uso reciente, son herramientas que ofrecen mejores opciones de reconstrucción para revisión de ATC y sobre todo optimizan el pronóstico a mediano y largo plazo, por tanto deberán incorporarse a la cirugía de revisión para evitar el cementado de componentes en la mayoría de los casos.

\section{BIBLIOGRAFÍA}

1. Urban RM, Hall DJ, Della Valle C, Wimmer MA, Jacobs JJ, Galante JO. Successful long-term fixation and progression of osteolysis associated with first-generation cementless acetabular components retrieved post mortem. J Bone Joint Surg Am. 2012; 94-A: 1877-1885.

2. Kim YH, Park JW, Patel C, Kim DY. Polyethylene wear and osteolysis after cementless total hip arthroplasty with alumina-on-highly cross-linked polyetlylene bearings in patients younger than thirty years of age. J Bone Joint Surg Am. 2013; 95: 1088-1093.

3. Howie DW, Neale SD, Martin W. Progression of periacetabular osteolytic lesions. J Bone Joint Surg Am. 2012; 94-A: e117.

4. Dallins JG, Math K, Scuderi GR. Evaluating the progression of osteolysis after total knee arthroplasty. J Am Acad Orthop Surg. 2015; 23: 173-180.

5. Lübbeke A, Garavaglia G, Barea C, Roussos C. Influence of obesity on femoral osteolysis five and ten years following total hip arthroplasty. J Bone Joint Surg Am. 2010; 92: 1964-1972.

6. Bozic K, Kurtz S, Lau E, Ong K, Vail TP, Berry DJ. The epidemiology of revision total hip arthroplasty in the united states. J Bone Joint Surg Am. 2009; 91A: 128-133.

7. Jacobs JJ, Roebuck KA, Archibeck M, Hallab NJ, Glant TT. Osteolysis: basic science. Clin Orthop Relat Res. 2001; 393: 71-77.

8. Archibeck M, Jacobs JJ, Roebuck K. The basic science of periprosthetic osteolysis. J Bone Joint Surg Am. 2000; 82-A: 1478-1489.

9. Sheth NP, Nelson CL, Springer BD, Fehring TK, Paprosky WG. Acetabular bone loss in revision total hip arthroplasty: evaluation and management. J Am Acad Orthop Surg. 2013; 21: 128-139.

10. Sheth NP, Rozell JC, Paprosky WG. Evaluation and treatment of patients with acetabular osteolysis after total hip arthroplasty. J Am Acad Orthop Surg. 2019; 27: e258-e267.

11. Tsukamoto M, Mori T, Ohnishi H, Uchida S, Sakai A. Highly cross-linked polyethylene reduces osteolysis incidence and wear-related reoperation rate in cementless total hip arthroplasty compared with conventional polyethylene at a mean 12-year follow-up. J Arthroplasty. 2017; 32 (12): 3771 3776.

12. Sheth NP, Nelson CL, Paprosky WG. Femoral bone loss in revision total hip arthroplasty: evaluation and management. J Am Acad Orthop Surg. 2013; 21: 601-612.

13. Patel AR, Sweeney P, Ochenjele G, Wixson R, Sturberg SD. Radiographically silent loosening of the acetabular component in hip Arthroplasty. Am J Orthop. 2015; 44: 406-410.

14. Adelani MA, Mall NA, Nyazee H, Clohisy JC, Barrack RL, Nunley RM. Revision total hip arthroplasty with retained acetabular component. J Bone Joint Surg Am. 2014; 96: 1015-1020.

15. Leung S, Naudie D, Kitamura N, Walde T, Engh CA. Computed tomography in the assessment of periacetabular osteolysis. J Bone Joint Surg Am. 2005; 87-A: 592-597.

16. Shohat N, Tan TL, Della Valle CJ, Calkins TE, George J, Higuera C, et al. Development and validation of an evidenced-based algorithm for diagnosing periprosthetic joint infection. J Arthroplasty. 2019; 34: 2730-2736.

17. Walmsley DW, Wadell JP, Schemitsch EH. Isolated head and liner exchange in revision hip arthroplasty. J Am Acad Orthop Surg. 2017; 25: 288-296.

18. Dattani R. Femoral osteolysis following total hip replacement. Postgrad Med J. 2007; 83: 312-316.

19. Bhandari M, Bajammmal S, Guyatt GH, Griffith L, Busse JW, et al. Effect of biphosphonates on periprosthetic bone mineral density after total joint arthroplasty. J Bone Joint Surg Am. 2005; 87-A: 293-301. 
20. Sköldenberg O, Rysinska A, Eisler T, Salemyr M, Bodén H, Muren O. Denosumab for treating periprosthetic osteolysis; study protocol for a randomized, double-blind, placebo-controlled trial. BMC Musculoskelet Disord. 2016; 17: 174.

21. Lachiewicz PF, Soileau ES. Highly cross-linked polyethylene provides decreased osteolysis and reoperation at minimum 10-year follow-up. J Arthroplasty. 2016; 31 (9): 1959-1962.

22. Saleh KJ, Thongtrangan I, Schwarz EM. Osteolysis: medical and surgical approaches. Clin Orthop Rel Res. 2004; 427: 138-147.

23. Su DH, Han SB, Yun HH, Chun SK, Shon WY. Characterization of progression of pelvic osteolysis after cementless total hip arthroplasty: Computed tomographic study. J Arthroplasty. 2013; 28: 1851-1855.

24. Lim SJ, Lee KH, Park SH, Park YS. Medium-term results of cementation of a highly cross-linked polyethylene liner into a well-fixed acetabular shell in revision hip arthroplasty. J Arthroplasty. 2014; 29: 634-637.

25. Rivkin G, Kandel L, Qutteinch B, Liebergall M, Mattan Y. Long term results of liner polyethylene cementation technique in revision for peri-acetabular osteolysis. J Arthroplasty. 2015; 30: 1041-1043.

26. Callaghan JJ, Liu SS, Phruetthiphat OA. The revision acetabulum-allograft and bone substitutes; vestigial organs for bone deficiency. Bone Joint J. 2014; 96-B (11 suppl A): 70-72.

27. Lachiewicz PF, Soileau ES, Martell JM. Wear and osteolysis of highly crosslinked polyethylene at 10 to 14 years: the effect of femoral head size. Clin Orthop Relat Res. 2016; 474: 365-371. 